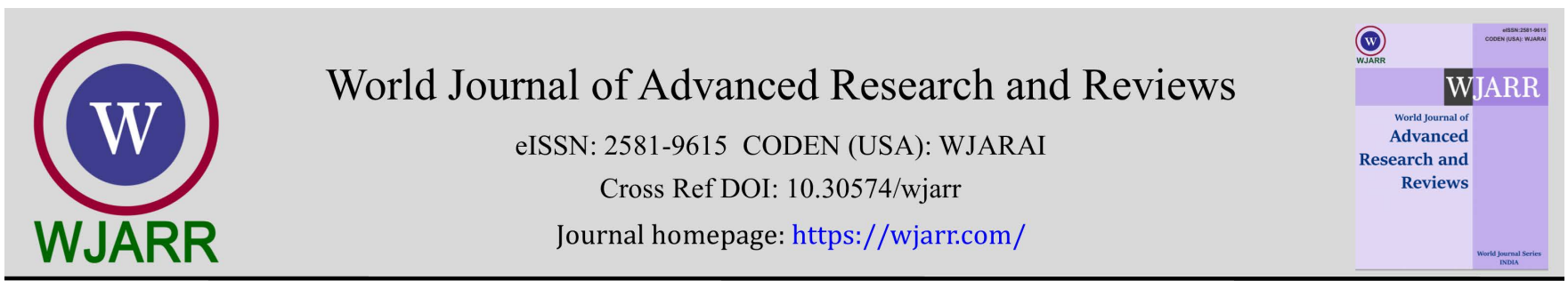

(RESEARCH ARTiClE)

\title{
Medical outcome and post-natal growth approach of premature infant in ambulatory follow-up in N'Djamena (Chad)
}

\author{
Kanezouné Gongnet *, Silé Souam Nguelé and Hikdjolbo Gonzabo \\ Department of pediatrics, Mother and Child Teaching Hospital, N'Djamena-Chad.
}

World Journal of Advanced Research and Reviews, 2021, 09(01), 256-261

Publication history: Received on 16 January 2021; revised on 23 January 2021; accepted on 25 January 2021

Article DOI: https://doi.org/10.30574/wjarr.2021.9.1.0028

\begin{abstract}
The survival of preterm infants depends on the health development level of each country and those who survive need a closer follow-up after the discharge. The objective of this study was to evaluate morbidity, mortality and post-natal growth of preterm infants in the first six-month of life in a low income country. This was a longitudinal study from May 2015 to June 2016 in the pediatrics department of the Mother and Child Teaching Hospital of N'Djamena. It involved all preterm infants hospitalized during six months of inclusion period and followed in this structure. The morbidity rate was $26.9 \%$ in the first month of follow-up. The more frequent pathologies were acute respiratory infections (37\%), functional digestive troubles (33\%) followed by anemia and malaria (15\%). Eight infants (11.9\%) had been hospitalized again with 3 deaths recorded (4.5\%). The growth was regular but the measures were mostly less than $-2 \mathrm{Z}$ score. Average weight was $6165.6 \mathrm{~g}$ at 6 months; average height: $60.72 \mathrm{~cm}$ and average head circumference: $41.62 \mathrm{~cm}$. Adjustment of cranial circumference was earliest with 53 and $87 \%$ of normal value at 3 and 6 months respectively. The improvement of care of preterm infants would need the development of perinatalogy and follow-up network.
\end{abstract}

Keywords: Prematurity; Follow-up; Morbidity; Mortality; Growth

\section{Introduction}

Premature baby is exposed, because of his immaturity, to numerous neonatal complications which condition his survival $[1,2]$. If the improvement of perinatal care makes it possible to take care of increasingly immature newborns and an improvement in survival at discharge, the question of the future of survivors remains open. Their evolution after returning home remains uncertain. Indeed, after their hospitalization, and mainly during their first year of life, premature infants should be monitored specifically because of the pathologies and multiple sequelae as reported by many European authors [3-6], but few data are available on the incidence of common pediatric pathologies in low income country like Chad. This assumes long-term medical care consumption [3- 6]. Long-term sequelae are important and the most to be feared [6-8].

The objective of this study was to evaluate the medical outcome and postnatal growth of premature babies during the first semester of life in N'Djamena in order to contribute to the improvement of care.

\footnotetext{
${ }^{*}$ Corresponding author: Kanezouné Gongnet; E-mail: kanezounegongnet@yahoo.fr

Department of pediatrics, Mother and Child Teaching Hospital, N'Djamena-Chad. 


\section{Materials and methods}

\subsection{Study design and population}

The study was conducted in the pediatrics' department of the Mother and Child Teaching Hospital of N'Djamena, the only structure specializing in the care of premature babies in the capital of Chad.

This was a longitudinal study conducted from 1st May 2015 to 30 June 2016. It involved all alive new-born less than 37 weeks of amenorrhea admitted to neonatal unit from May to October 2015. Premature babies who died on arrival, left against medical advice or escaped were excluded from the study. Only survivors at discharge were eligible for outpatient follow-up and data from regularly followed infants were taken into account in the analysis.

\subsection{Data collection}

Data were collected continuously during hospitalization and follow-up consultations on a pre-established form. On discharge from the hospital, a one-week appointment is given to the parents for follow-up. The frequency of consultations was one per week during the first month then one consultation every two weeks depending on the clinical condition and finally one consultation per month when the post-conceptual age reaches 40 weeks subject to a weight of at least $2500 \mathrm{~g}$ and clinically stable. The evaluation focused on the state of health, growth in height and weight and psychomotor development. A complete blood count was taken at 3 months and then at 6 months of postnatal age. The search for Plasmodium was done on symptom appeal.

Data collected concerned neonatal characteristics (sex, gestational age, measurements, trophicity, complications), follow-up parameters (morbidity, measurements, rehospitalization and mortality). Outcome was assessed in terms of morbidity, mortality and postnatal growth.

\subsection{Patient consent statement}

Informed parental consent was obtained and the data collected was kept confidential.

\subsection{Statistical analysis}

Data entry and analysis were performed using SPSS 20 software. The Chi-square test (or Fisher's exact test) was used for the comparison of proportions with a significance level of $5 \%$.

\section{Results}

\subsection{Characteristics of study population}

The flow diagram of premature babies followed-up is showed on figure 1. Premature babies followed-up after returning home were divided into medium premature $(34.3 \%)$, very premature $(62.7 \%)$ and extreme premature (3\%). They were hypotrophic in $10.6 \%$ of cases. The sex-ratio was 1.03 . The average weight was $1410 \mathrm{~g}$, average height $40 \mathrm{~cm}$, and average head circumference $28.76 \mathrm{~cm}$. Among neonatal complications, respiratory distress was found in $43.3 \%$ of cases, infections in $31.4 \%$ of cases and anemia in $23.9 \%$ of cases.

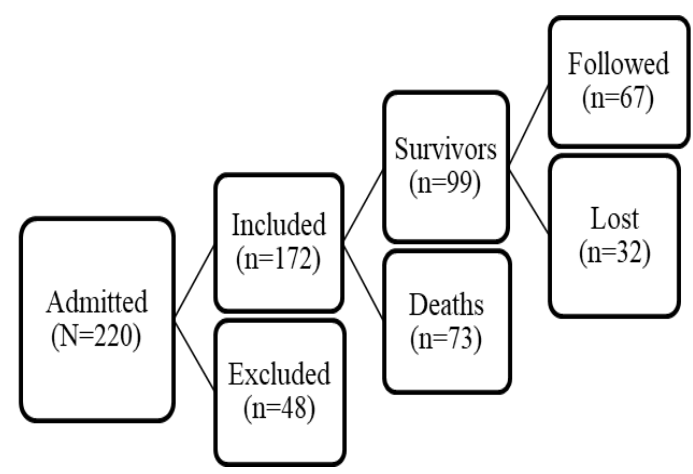

Figure 1 Flow diagram of premature babies. 


\subsection{Morbidity during follow-up}

The morbidity rate during the month after returning home was $26.9 \%$. The main diseases and disorders recorded during the first six-month of life are grouped in Table I.

Table I incidence of pathologies and disorders during follow-up.

\begin{tabular}{|l|l|l|}
\hline Pathologies & $\mathbf{n}$ & $\mathbf{\%}$ \\
\hline Acute respiratory infections & 25 & 37.3 \\
\hline Functional digestive disorders & 22 & 32.8 \\
\hline Dermatosis & 9 & 13.4 \\
\hline Abdominal hernias & 5 & 7.5 \\
\hline Anemia & 10 & 14.9 \\
\hline Malaria & 10 & 14.9 \\
\hline Conjunctivitis & 8 & 11.9 \\
\hline Oral candidiasis & 3 & 4.5 \\
\hline Dehydratation & 1 & 1.5 \\
\hline
\end{tabular}

Digestive disorders were more frequent in the first trimester (32.8\%) compared to the second trimester (4.5\%). The frequency of functional digestive disorders was $52.2 \%$ in medium premature babies and $23.8 \%$ in very premature babies $(\mathrm{p}=0.04)$. Hypotrophs at birth developed more acute respiratory infections (85.7\% vs. 32.2\%; $\mathrm{p}=0.010)$, dermatosis (42.9\% vs. 10.2\%; $p=0.048)$ and abdominal hernias (42,9\% vs. 3.4\%; $p=0.007)$. Anemia was more common during follow-up in premature infants with neonatal infection $(38.1 \%$ vs. $4.3 \%$; $=0.000)$. Eight premature babies (11.9\%) were rehospitalized for decompensated anemia ( 5 cases; malaria was associated in 4 cases), acute respiratory infections associated with malaria ( 2 cases) and late neonatal infection (1 case).

\subsection{Mortality during follow-up}

Three deaths were recorded for a death rate of 4.5\%. These were all premature babies from 34 weeks, including 2 male and one female. Causes of death were severe malaria (1) and severe acute respiratory infections (2).

\subsection{Post-natal growth}

The average weight was $3951.6 \pm 1009.5 \mathrm{~g}$ at 3 months and $6165.6 \pm 1252.2 \mathrm{~g}$ at 6 months. The average height was $51.36 \pm 5.2 \mathrm{~cm}$ at 3 months and $60.72 \pm 4.63 \mathrm{~cm}$ at 6 months. The average head circumference was $37.91 \pm 2.37 \mathrm{~cm}$ at 3 months and $41.62 \pm 1.69 \mathrm{~cm}$ at 6 months. Six infants (17.6\%) were normal weight for age at 3 months and 13 (41.9\%) were at 6 months; Seven (20.6\%) and 11 (35.5\%) were normal height for age at 3 and 6 months, respectively; 18 $(52.9 \%)$ and $27(87.1 \%)$ had normal head circumference for age at 3 and 6 months, respectively. The growth rate of average measurements was : $26.42 \mathrm{~g} /$ day (28.24 g/day in the 1 st trimester and $24.6 \mathrm{~g} /$ day in the second) for weight; $3.44 \mathrm{~cm} /$ month $(3.67 \mathrm{~cm}$ in the 1 st trimester and 3.12 in the second) for the size and $2.14 \mathrm{~cm} / \mathrm{month}$ ( $3.05 \mathrm{~cm}$ in the $1 \mathrm{st}$ trimester and $1.24 \mathrm{~cm} /$ month in the second) for the cranial perimeter. The growth curves for mean weight (figure 2), mean height (figure 3) and cranial perimeter (figure 4) are below. 


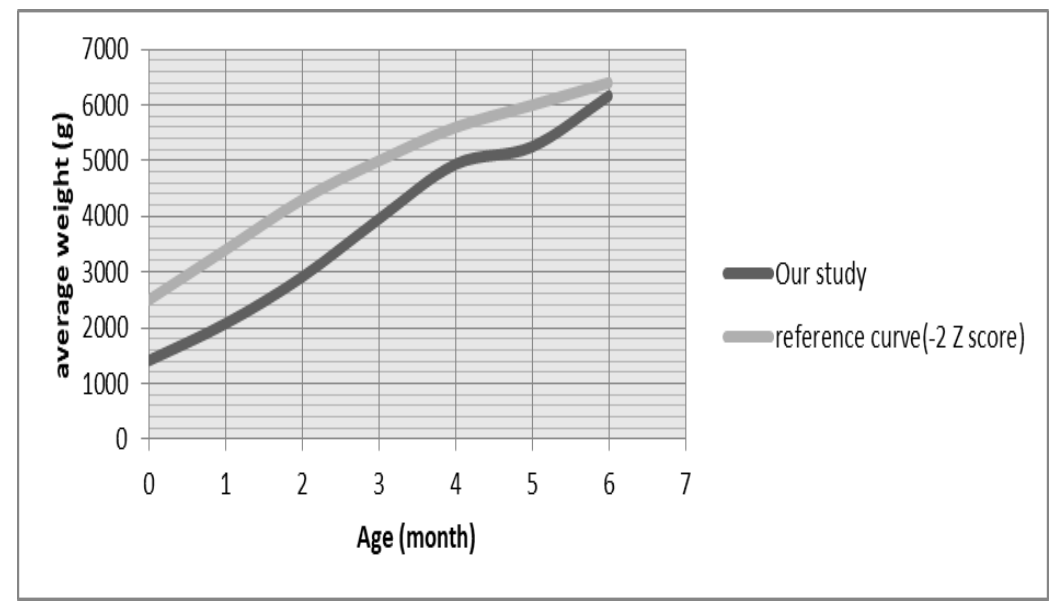

Figure 2 change in average weight during the first semester

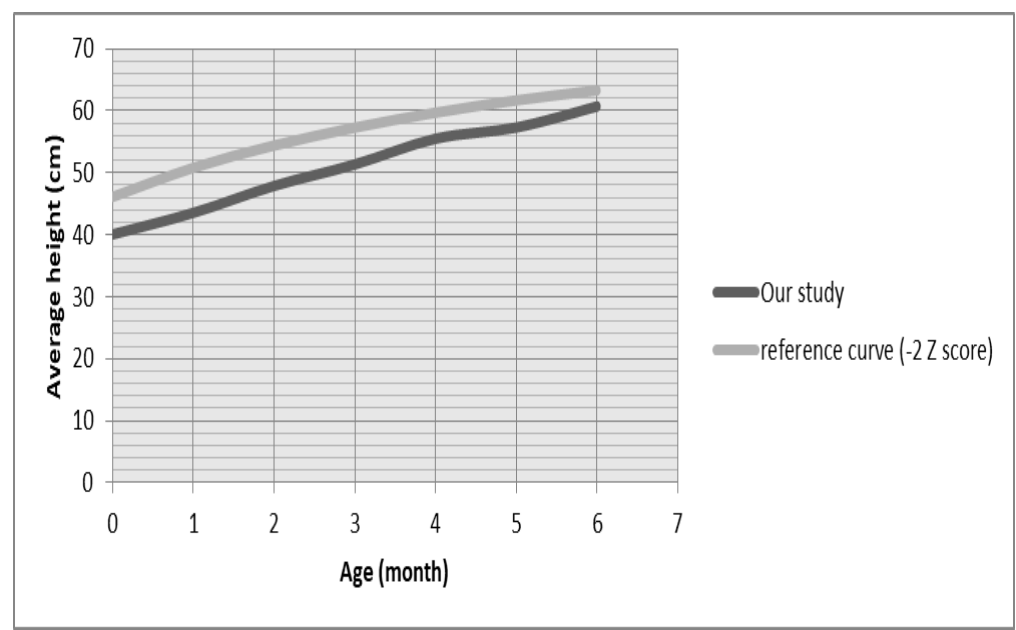

Figure 3 change in average height during the first semester

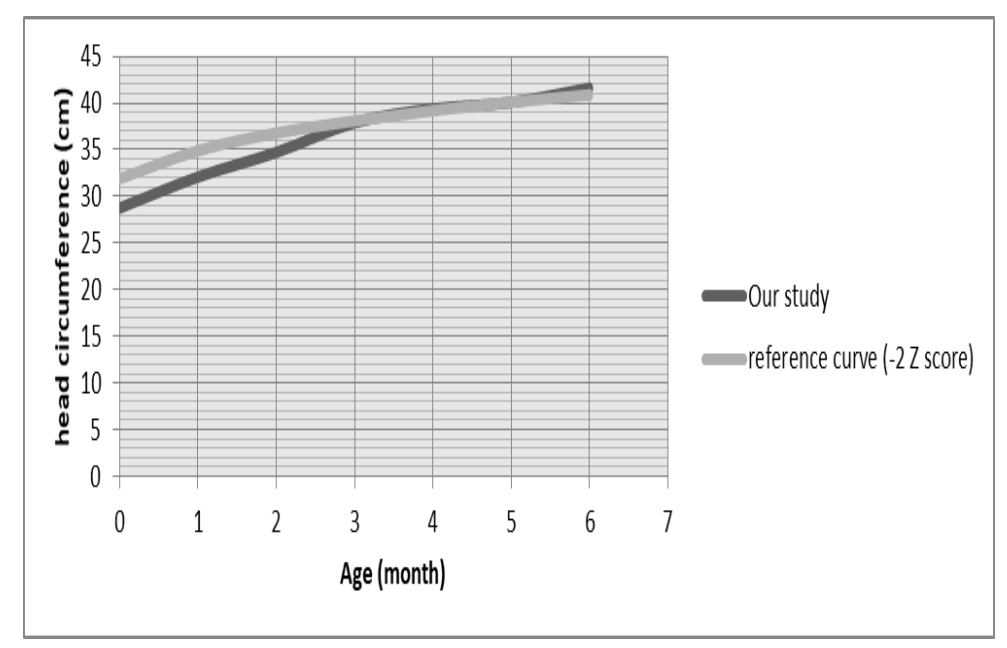

Figure 4 change in average head circumference during the first semester 


\section{Discussion}

The aim of this study was to assess morbidity, mortality and post-natal growth of premature infants after the discharge in the first semester of life in N'Djamena, Chad, a low income country.

The first main outcome of this study is that morbidity is significant during the first semester and the disorders observed are responsible for rehospitalization and a significant mortality. We found a $26.9 \%$ morbidity rate during the month following discharge from hospital; which allows us to say that the first weeks after returning home are critical and decisive for the subsequent development of the premature baby. More rigorous follow-up including even home visits should be done during this period. Morbidity during follow-up was dominated by acute respiratory infections, functional digestive disorders, anemia and malaria as reported by DAINGUY and al [9]. However, abdominal hernias, skin conditions and conjunctivitis are not uncommon. Digestive disorders were much more frequent in the first trimester compared to the second. The frequency of acute respiratory infections remained unchanged during the semester. It was also noted a predominance of digestive disorders when gestational age is more advanced. The method of breastfeeding and the hygiene associated with it could be explanatory factors since they differ from one mother to another. Acute respiratory infections, dermatosis and abdominal hernias were more common when hypotrophy is associated with prematurity. The occurrence of anemia was linked to existence of a neonatal infection.

The various pathologies led to rehospitalizations of premature infants in $12 \%$ of cases and mainly for decompensated anemia. DAINGUY and al in Ivory Coast found a rehospitalization rate of $30 \%$ with the main reasons for acute respiratory infections and anemia [9]. Acute respiratory infections are also retained as the main reason for rehospitalization by BLONDEL and al in France who had found a rehospitalization rate of nearly $40 \%$ [5]. The much lower rate of rehospitalization in the present study could be explained by the early management of the pathologies presented by the infants while the symptoms are less marked, thus avoiding a new hospital stay. All the rehospitalizations having taken place in the first trimester, it is therefore important that the premature baby be followed at a regular and closer pace during this period.

Among the rehospitalized premature babies, 3 died representing $4.5 \%$ of the survivors who benefited from longitudinal follow-up.

The second main outcome of this study is that growth and weight development of premature infants is regular, measurements are mostly inferior to $-2 \mathrm{Z}$ score in the first semester. Our study showed that growth rate of the various parameters is higher than standard norms. Adjustment is faster for the cranial circumference with $87 \%$ of normal value at 6 months in our study. Its growth is faster in the first trimester with twice the speed than in the second trimester. The particularity of the growth of premature babies has also been reported by DAINGUY and al in Ivory Coast and by DIAGNE in Senegal $[9,10]$. Diagne and al also noted that premature babies had good growth dynamics especially in the first semester and a more marked delay in the acquisition of a normal weight for age compared to the two other parameters. Moreover, growth was independent of gestational age and trophicity at birth.

Our study has some limitations. Firstly, 32.3\% of premature babies released alive were lost to follow-up. This leads us to say that mortality rate is probably underestimated. However, it should be emphasized that the morbidity and mortality of premature infants can no longer be assessed in the neonatal period alone. This high rate of lost to followup could be explained by the low level of education of mothers, socio-economic reasons, geographic reasons, and the lack of awareness of the risks of prematurity. Interruption of follow-up is justified by some mothers by the good health of the child, so they do not find it beneficial to bring a healthy child for consultation. Houenou and al, Dainguy and al in Ivory Coast showed in their study that the couple's socio-economic instability and lack of awareness were major obstacles to follow-up $[9,11]$.

The second limitation is the shorter duration of our study that made the correction for weight and height could not be really appreciated. The second semester of life, during which food diversification is initiated, would have made it possible to assess the impact on the growth of premature babies. A long-term study is needed to assess not only growth in height and weight but also psychomotor development and long-term sequelae.

\section{Conclusion}

Morbidity is significant after the return home of the premature infant, dominated by acute respiratory infections, functional digestive disorders and anemia. Disorders observed are responsible for rehospitalization and a significant mortality. The first trimester of life is crucial for the subsequent development of the premature baby, hence the need 
for regular monitoring during this period. The growth of the premature infant is regular, independent of the degree of prematurity and trophicity at birth, and follows different standards from that of the term newborn. The correction of the cranial circumference is early. A long-term study at least at preschool age would make it possible to properly assess the development of premature babies and to detect the inherent sequelae in the African context. Improving the care of premature babies would require the development of a perinatology and follow-up network.

\section{Compliance with ethical standards}

\section{Acknowledgments}

We Acknowledge the Director of the Mother and Child Teaching Hospital of N'Djamena.

\section{Disclosure of conflict of interest}

There are no conflicts of interest in connection with this paper.

\section{Statement of informed consent}

Informed parental consent was obtained for all participants included in this study.

\section{References}

[1] Magny JF, V oyer M, F Kieffer, Coatantiec Y. Prematurity. Encycl Méd Chir (Elsevier, Paris), Practical Encyclopedia of Medicine. 1998; 8-0320.

[2] Howson CP, Kinney MV, Lawn JE. Born too soon: The Global Action Report on Preterm Birth. Geneva: March of Dimes, PMNCH, Save the Children, WHO. 2012.

[3] Zupan-Simunek V. Outcome of premature babies in 2008 in France. Clamart: Antoine Béclère Hospital. 2008.

[4] Zupan-Simunek V. Organization of monitoring of very premature babies: the role of city-hospital networks. Pediatric Therapeutic Medicine 2004; 7(4): 287-96.

[5] Blondel B, Truffert P, Lamarche- $V$ adel A, Dehan M, Larroque B. Use of medical services by adults during the first year of life in the EPIP AGE cohort. Arch Pediatr. 2003; 10: 960-8.

[6] Ancel PY, Breart G, Kamiski M, Larroque B and the EPIP AGE group. Outcome of very premature children at school age. BEH 16-17/ 4 May 2010; 198-200.

[7] Larroque B, Delobel M, Arnaud C, Marchand M and the EPIP AGE Group. Become very premature children at 5 and 8 years old . Arch Pediatr. 2008; 15: 589-91.

[8] Larroque B, Ancel PY, Marret S, Marchand L, André M, Arnaud C, et al; EPIP AGE Study group. Neurodevelopmental disabilities and special care of 5-year-old children born before 33 weeks of gestation (the EPIPAGE study): a longitudinal cohort study. Lancet. 2008; 371(9615): 813-20.

[9] Dainguy ME, Folquet AM, Akaffou E, Sylla M, Kouadio E, Kouakou C, Houenou Y. Outpatient follow-up of premature babies during the first year of life in the pediatric department of the CHU of Cocody. Mali Med. 2011; 26 (3): 25-9.

[10] Diagne NR. Perinatal characteristics and outcome of premature infants: about 277 cases. [Thesis Med]. Dakar: Cheik Anta Diop University (FMPOS); 2000.

[11] Houenou AY, Ecra JF, Folquet AM. Surveillance and health promotion of premature babies aged 0 to 1: Benefits and obstacles. Ann Pediatr. 1999; 46(10): 730-6. 